\title{
Insights into the Mechanisms of action of Chloroquine and Hydroxychloroquine and its use in COVID-19 for chemoprophylaxis
}

monalisa sahu ${ }^{1}$, Arvind Kumar ${ }^{1}$, Sujay Halkur Shankar ${ }^{1}$, Vishal Vishwakarma ${ }^{1}$, Pradipt $\mathrm{Sahoo}^{2}$, and Naveet Wig${ }^{1}$

${ }^{1}$ All India Institute of Medical Sciences

${ }^{2}$ Kalinga Institute of Medical Sciences

May 18, 2020

\begin{abstract}
The global outbreak of Coronavirus disease 2019 (COVID-19), caused by the Severe Acute Respiratory Syndrome CoronaVirus 2 (SARS-CoV-2/2019-nCoV) has put forth a serious threat to the international public health and has ruined the global economy. Till date, no drugs have been approved for Coronavirus Disease-2019 (COVID-19), although use of some drugs in trial phase has been attempted. The drugs being used for management of COVID-19 disease include chloroquine (CQ), hydroxychloroquine (HCQ), and remdesivir. In this article, we have aimed to review existing literature and mechanism by which CQ and HCQ have an effect on COVID-19, most importantly by interfering with autophagy, lysosomal activity, receptor binding and membrane fusion. We have systematically searched the PubMed database upto April, 2020 and analysed all the articles published on CQ, HCQ and COVID-19. The available data provides insights into the immunomodulatory potency of HCQ, along with the molecular mechanism of action of the drug on the SARS-CoV-2.
\end{abstract}

\section{Introduction}

As of May14, 2020, over 4,218,212 people have been affected by the COVID19 across the globe and it has claimed 290,242 lives. Billions of people across the world are under serious threat of the disease, with as many as 215 nations or territories being under its clutches. Billions of Indians are currently at risk of acquiring COVID-19 [1]. Although India showed a staggered course of SARS-CoV-2 transmission, with 18,539 cases diagnosed between Jan 31, and April 21, 2020, however, there has been a recent surge in cases, with the numbers rising as of end of April and the mid of May to about 78,003. [2]. The Indian Council of Medical Research (ICMR), under the Ministry of Health and Family Welfare (MOHFW), has proposed for chemoprophylaxis with HCQ at a dose of $400 \mathrm{mg}$ twice on day 1 and $400 \mathrm{mg}$ once a week thereafter for asymptomatic medical and healthcare workers (HCWs) who are involved in the treatment of patients with confirmed or suspected COVID-19, and for asymptomatic household contacts of the confirmed cases [3]. The record mentions that the utilization of HCQ in prophylaxis has been derived from the accessible proof of benefit as treatment and has been further upheld by the available preclinical data.

There are some in vitro studies, which demonstrate the antiviral action of HCQ and its precursor CQ, but there is lack of much peer-reviewed data assessing the use of either drug for exposure prophylaxis of SARSCoV-2 infection. For the treatment of diagnosed cases, a few studies have been published, one of which demonstrates faster reduction of nasopharyngeal viral carriage, without any information on clinical improvement [4], where as the other study was inconclusive about the beneficial effects of HCQ due to the smaller sample size [5].

Coronaviruses (CoVs) are enveloped positive sense RNA viruses, which range from $60 \mathrm{~nm}$ to $140 \mathrm{~nm}$ in diameter. It has spike like projections on its surface, having a crown like appearance on the electron 
microscope, hence named coronaviruses. The virus belongs to the family Coronaviridae, order Nidovirales, and has been classified into three genera: alphaCoVs, betaCoVs, and deltaCoVs. In the last two decades, two of the animal betaCoVs have crossed over to the humans causing severe diseases, which include Severe Acute Respiratory Syndrome CoronaVirus (SARS-CoV), and Middle East Respiratory Syndrome CoronaVirus (MERS-CoV); responsible for severe acute respiratory disease outbreak in China in 2002-2003 [6] and in the Middle East in 2012, respectively [7].

Novel coronavirus, officially known as SARS-CoV-2, the etiological agent of the COVID-19, emerged in Wuhan, Hubei province, China. On 11th March 2020, the World Health Organization (WHO) reported this disease as pandemic [8].

The clinical features of COVID-19 are varied, and range from asymptomatic infection to acute respiratory distress syndrome, and multi-organ dysfunction. The common clinical features include mainly the fever, cough and pneumonia. Sequence identity of about $80 \%$ is seen between SARS-Cov-2 and SARS CoV; and a $96.2 \%$ sequence identity with BatCoV RaTG13, a bat CoV [9]. Under the circumstances of lack of clinically efficient treatment and a "public health emergency", several attempts are being made using numerous drugs for the treatment of COVID-19. The drugs under trial include a minimum cost antimalarial drug like chloroquine (CQ), its derivative hydroxychloroquine (HCQ), a nucleotide with broad spectrum antiviral activity, remdesivir and combination anti-retroviral drug lopinavir/ritonavir.

During the previous SARS outbreak, HCQ was detected to have anti-SARS-CoV activity in vitro [10]. Thus, HCQ has attracted the attention of many treating physicians over the last few weeks, as a drug with the potential for management of cases of COVID-19. Several reports have recommended that the drug could be used for the treatment of COVID-19, inspite of the fact that the clinical effectiveness of these medications has not yet been completely assessed.

HCQ use has become a part of recent treatment guidelines for rheumatoid arthritis (RA), antiphospholipid syndrome (APS), systemic lupus Erythematosus (SLE) and primary Sjogren syndrome [11].

The observational studies conducted demonstrating the use of antimalarial drugs in SLE, with particular emphasis on HCQ, have found definitive evidence that this molecule possesses an immunomodulatory activity, thus enhancing the long-term survival in the SLE patients. It also has the potential to manage autoimmune disease activity during pregnancies without evidence of any embryotoxic or fetotoxic effects. There is evidence suggesting that HCQ can prevent incidence of organ injury, including bone destruction [12]; modify autoimmunity [13], and possesses antithrombotic effects [14]. Moreover, HCQ has fewer concerns regarding drug- drug interactions.

\section{Mechanisms of action}

The exact molecular mechanisms of action of CQ and HCQ have not been completely elucidated. Various modes of action have been proposed by which CQ and HCQ exerts its therapeutic action, by inhibiting the coronavirus at a series of steps, although, majority of them have been on in vitro experiments. The link between these mechanisms of actions, the safety profile observed and the clinical efficacy obtainedin vivo have yet to be fully delineated.

The main mode of action of CQ and HCQ is the interference with autophagy, lysosomal activity, receptor binding and membrane fusion. It has been widely acknowledged that CQ and HCQ accumulate in lysosomes (lysosomotropism) and inhibit their working.

\section{Autophagy}

Autophagy is defined as a conserved transport pathway, wherein targeted structures get sequestered by phagophores, subsequently maturing into autophagosomes, and then being further delivered into the lysosomes for degradation. The process of autophagy is involved in the pathophysiology of several diseases and its modulation has proven beneficial in the outcome of numerous specific diseases [15]. CQ and HCQ are lysosomal inhibitors, and have been used to block autophagy in in vitro experiments, with the assumption 
that they primarily block lysosomal degradation. CQ and HCQ are FDA-approved drugs for use in clinical trials aimed to treat tumors through autophagy inhibition, although the exact mechanism by which CQ blocks autophagy yet remains to be firmly demonstrated.

Impairment of autophagosome fusion with lysosomes and blockade of autophagic flux, thus inhibiting autophagy, contributes to the main mechanism of CQ and HCQ action. The morphology of degradative compartments of the cell are affected differently by CQ and HCQ as compared to the other lysosomal inhibitors. The Golgi complex and the endolysosomal system are disorganized both in vitro and in vivo as well as endosomal trafficking are affected by CQ and HCQ.

Alongwith alteration of its organization, the drugs also affect the functionality of the endolysosomal system. Prevention of co-localization of virions with early endosomes (EEs) or endolysosomes (ELs) is caused by CQ and HCQ. This implicates that both CQ and HCQ block the transport of SARS-CoV-2 from EEs to ELs, which is required for the release the viral genome as in the case of SARS-CoV. Moreover, both CQ and HCQ treatment cause observable changes in the number and morphology of EEs and ELs. In the untreated cells, most EEs were more compact than ELs, where as in the CQ and HCQ-treated cells, abnormally enlarged EE vesicles were seen, inside which the virions were localized.

In addition, HCQ also induces an autophagy-independent severe disorganization of the Golgi complex and EL, which could possibly contribute to the fusion impairment. This has been demonstrated in the HCQ treated mice, which showed severe alteration in the Golgi and the EL system in an autophagy independent manner [16].

\section{Interference with Lysosomal activity}

CQ and HCQ also interfere with lysosomal activity. Lysosomes contain hydrolytic enzymes, and its main functions include assistance of different vesicles in autophagy (digestion of cargo such as cell organelles and material that are inside the cell) and phagocytosis or endocytosis (digestion of material from outside the cell).

Lysosomes are involved not only in recycling cellular substrates, but also in antigen processing and MHC class II antigen presentation, thus indirectly modulating immune activation. HCQ primarily increases the intracellular $\mathrm{pH}$, thus inhibiting lysosomal activity in antigen presenting cells (APCs), which include plasmacytoid dendritic cells (pDCs) and B cells. It further prevents antigen processing and major histocompatibility complex (MHC) class II-mediated autoantigen presentation to T cells [17]. This consequently results in reduced $\mathrm{T}$ cell activation, and lesser differentiation and expression of co-stimulatory proteins such as CD154 on CD4T cells [18]. It also results in decreased cytokines production by $\mathrm{T}$ cells and B cells such as IL-1, IL-6 and TNF [19]. The flowchart detailing the immunomodulatory mechanism of HCQ is shown in Figure 1.

Lysosomal proteases also activate the fusion process between host and viral membranes by cleaving coronavirus surface spike (S) proteins [20]. The virally enoded S protein is involved in the process of endocytic viral entry into the host cells, mediated by its proteolytic cleavage to form two subunits, S1 and S2 [21]. Increase in the $\mathrm{pH}$ of the lysosome by HCQ prevents lysosomal protease activity, thus disrupting the fusion process [22]. In the absence of the $\mathrm{pH}$ necessary for the executing the endosome and lysosome cleavage function, replication of and infection by the coronavirus are obliterated [23]. This led to the inhibition of SARS-CoV spread in cells treated with CQ prior to or after infection, thus highlighting both prophylactic as well as therapeutic advantages of CQ in combating SARS-CoV [24].

\section{Interference with Autophagy and lysosomal activity}

Autophagy as well as interference of lysosomal activity has been associated with immune activation and antigen presentation [25]. Anin vitro study suggested that autophagy has a significant role in MHC class II-mediated autoantigen presentation by antigen-presenting cells to CD4+ T cells [26]. The $\mathrm{pH}$ of the lysosomes, which is perfect for the lysosomal enzymes involved in hydrolysis, is increased in the endosomal compartments by CQ and HCQ. This results in weakened autophagosomes, interference with lysosomal maturation and function; and subsequently inhibition of antigen presentation and immune activation. Researches 
are underway to determine the main molecular targets of CQ and HCQ. Palmitoyl-protein thioesterase 1 (PPT1), an enzyme involved in the catabolism of lipid-modified proteins, has been identified as a potential lysosomal target of CQ and HCQ. The drugs get attached to it and inhibit PPT1 activity [27]. Further detailed studies are nevertheless warranted for the identification and confirmatory functional studies on other molecular targets within the lysosomes. The graphical representation of these mechanisms have been depicted in figure 2.

\section{Implication of autophagy in CoVs infection}

In the past one and a half decades, the implication of autophagy in CoV infection has attracted the attention of many researchers. It was probably due to the developing field of autophagy research and the SARS outbreak across the world in 2002-2003, both occurring around the same time.

According to the currently ongoing researches, different reports have converged onto two important questions: as to whether $\mathrm{CoV}$ prompts autophagy and whether the autophagy machinery or Autophagy related (ATG) proteins are involved in the infection by the CoVs and their replication.

The first report demonstrating the role of autophagy in viral replication was based on the study of coronavirus Mouse Hepatitis Virus (MHV) [28]. The authors made some important observations in this study. They observed that infection with MHV was responsible for the formation of double-membrane vesicles (DMVs). RNA replication of the MHV occurs on these DMVs, inside the host cell cytoplasm. Cellular autophagy as been proposed as the mechanism by which the replication complex formation occurs in positive sense RNA viruses, in the form of DMVs. This suggests an autophagic origin of the DMVs.

The markers for autophagic vacuoles on mammalian cells have been identified as microtubule-associated protein light chain 3 (LC3) and Apg12. The viral replication complexes on DMVs were observed to be colocalized with the LC3, Apg12 and other autophagy proteins, suggesting the role of autophagy in the viral replication. In their experiment, they also observed that MHV replication was impeded in APG5 knockout mice. Thus, the authors concluded that autophagy plays a major role in the formation of DMV and the efficiency of replication of MHV is greatly enhanced by the formation of DMV [28]

In a subsequent report by the same group, in SARS-CoVs, a similar co-localization of the key viral replicase proteins with nsp and endogenous LC3, a protein marker for autophagosome, was observed [29].It thus provided evidence for the similar contribution of autophagy in the replication of SARS-CoVs like for the MHV.

Cottam et al used a avian coronavirus, Infectious Bronchitis Virus (IBV) and detected that one of the key viral replication protein, nsp6, is capable of activating autophagy [30]. Notably, this nsp6 is also present in SARS-CoV and MHV. Thus, it would be of interest to further test the effects of nsp6 on autophagy, in these two CoVs.

On the contrary, a few studies failed to demonstrate the association between autophagy and CoV infection and replication. In a study conducted by Snijder et al in Vero cells infected with SARS-CoVs, they failed to detect the co-localization of GFP-LC3 or LC3 with the viral replication-transcription complexes in SARS$\mathrm{CoV}$. They utilized immunofluorescence staining to carry out the analysis [31].

\section{Involvement of the endocytic pathway in CoVs infection}

Entry of the virus into the host cells is one of the key deciding variables in viral infection. At present, it is broadly believed that the entry of the CoVs into the host cells occurs primarily through two routes, namely the endosomal/ endocytic pathway and the cell surface non-endosomal pathway, the former being more contributory and has been extensively studied [32].

CoVs are enveloped, positive-strand RNA viruses and contain a set of four proteins that encapsidate the viral genomic RNA: the nucleocapsid protein $(\mathrm{N})$, the membrane glycoprotein $(\mathrm{M})$, the envelope protein $(\mathrm{E})$ and the spike glycoprotein (S) [33]. The S protein is the key protein involved in the process of endocytic viral entry into the host cells, and is mediated by its proteolytic cleavage to form two subunits, S1 and S2 
[21]. S1 is responsible for receptor-binding, whereas $\mathrm{S} 2$ is mainly for membrane fusion by formation of a extended fusion platform. It thus leads to the infection of the host cells with the virus.

HCQ and CQ inhibit receptor binding and membrane fusion, which comprise the two key steps involved in the entry of coronaviruses into the cell. Antiviral effects of CQ and HCQ have been demonstrated during pre- and post-infection conditions by interference with the glycosylation of angiotensin-converting enzyme 2 (ACE2), the cellular receptor of SARS-CoV, and thus preventing virus fusion with the host cell. Impairment of terminal glycosylation of ACE2 may result in reduction of the binding efficiency between ACE2 located on host cells and the SARS-CoV spike (S) protein. Consequently, the binding of the virus to the receptors on the cell surface is largely impeded and thus, the infection is prevented.

Acidification is a crucial step for the endosome maturation and function, and, CQ and HCQ raise the pH. In presence of $\mathrm{CQ}$ and $\mathrm{HCQ}$, endosome maturation might be occluded at the intermediate stages of endocytosis, culminating in the failure of further transport of virions to the final releasing site.

Following administration of HCQ and CQ, the drugs enter the cell and are both concentrated inside organelles having low $\mathrm{pH}$, like the endosomes, Golgi vesicles and lysosomes. As endosomes are used as the primary cellular entry mechanism by the viruses, a rise in the $\mathrm{pH}$ of endosomes following HCQ treatment has a negative influence on the the process of fusion between the virus and endosome [24].

The main report which demonstrated the significance of endosome-lysosome in CoVs was the morphological report using electron microscopy, in which two CoVs, (IBV and PEDV) were used. Virus-containing electrondense cytoplasmic bodies were detected in late stages of infection with these viruses, and they probably represented lysosomes accumulating viral particles [34]. This provides evidence for the possible functional implication of lysosome in CoVs infection.

\section{Safety profile and Pharmacokinetics of CQ and HCQ}

CQ and HCQ efficiently inhibit SARS-CoV-2 infection in vitro . Easy availability, proven safety profile, and a relatively lower cost of the drugs further add to the ease of these drugs being considered as therapeutic options for management. HCQ has been demonstrated to be much less ( $40 \%)$ toxic than CQ in animals. More importantly, HCQ is still available very widely for the treatment of autoimmune diseases, such as systemic lupus erythematosus and rheumatoid arthritis. The chemical structures and mechanisms of both the drugs is similar, acting as a weak base and an immunomodulator.

CQ and HCQ have been reported to have very efficient oral absorption in humans. Their drug levels in lungs are almost 200- 700 times higher than that in the plasma. The safe dosage of HCQ sulfate is 6- $6.5 \mathrm{mg} / \mathrm{kg}$ per day, which could produce serum levels of 1.4- 1.5 $\mu \mathrm{M}$ in humans. The high levels achieved in the tissues is sufficient to inhibit SARS-CoV-2 infection [35].

Clinical investigations have revealed that cytokine storm has been associated with disease severity [36]. Along with the direct antiviral activity, HCQ also has a good safety profile and is a successful anti-inflammatory agent, used extensively in autoimmune diseases and significantly decreases the production of cytokines and pro-inflammatory factors. Hence, in COVID-19 patients, HCQ may also prove beneficial by attenuating the inflammatory response.

\section{Insights into use of CQ and HCQ in COVID-19 for chemoprophylaxis}

There have been very few in vivo studies demonstrating the use of HCQ as chemoprophylactic agent. Lee et al conducted an in vivostudy in Korea [37], where they administered HCQ as post-exposure prophylaxis (PEP) for COVID-19 in 211 persons including 189 patients and 22 health care workers (HCWs) in a long term care hospital. Initially, all these exposed individuals were negative for SARS CoV2 by PCR. PEP with HCQ at a dose of $400 \mathrm{mg}$ per day for 14 days was completed in 184 patients and $21 \mathrm{HCWs}$ without any serious adverse outcomes. After the completion of 14 days quarantine period and medication, the follow-up PCR in all the individuals were negative. This is the first study conducted using HCQ in PEP, as there are no existing guidelines for PEP. Although this was a single centre study, and there was no adequate control 
group, the outstanding results prompt the use of HCQ as PEP in settings where there are chances of high risk exposure, such as the asymptomatic HCWs involved in care of suspected or confirmed cases of COVID-19 and asymptomatic household contacts of laboratory confirmed cases.

Offering HCQ as a pre-exposure prophylaxis to the HCWs providing care to the COVID-19 patients, and asymptomatic household contacts of confirmed positive cases, who always stand a chance of high risk exposure, may prove to be a useful strategy in preventing the spread of the disease. The ICMR guidelines also somewhat hint at a similar strategy. All of this can be undertaken, keeping in mind the adverse outcomes and safety profile of the drug.

\section{Conclusion}

In conclusion, experiments and data show that CQ and HCQ can efficiently inhibit SARS-CoV-2 infection in vitro, with $\mathrm{HCQ}$ being better than $\mathrm{CQ}$ in several respects. Intracellular mechanism of action and anti-inflammatory function together prove the drug to have a good potential to combat the disease, but this possibility requires further confirmation by clinical trials. HCQ may be considered for pre- and postexposure prophylaxis in individuals with high risk exposure. It is noteworthy that, although the toxicity of HCQ is less than CQ, prolonged usage and overdose of HCQ can still cause poisoning. The relatively lower safety index of HCQ warrants conducting well planned and structured clinical trials for achieving efficient and timely control of the SARS-CoV-2 infection.

Hence, undertaking well planned and careful in vitro and in vivo testing is essential for providing definitive evidence for the use of HCQ in COVID-19 prevention and disease progression.

\section{COMPETING INTERESTS}

The authors report no conflict of interest

\section{ACKNOWLEDGEMENT}

The figures were made using BioRender.com.

\section{References}

1. World Health Organization. Situation reports. Available at: https:// www.who.int/emergencies/diseases/novel-coronavirus-2019/ situation-reports/. Accessed 14 May 2020 .

2. Ministry of Health and Family Welfare. https://www.mohfw.gov.in. Accessed on 14 May 2020.

3. Indian Council of Medical Research [Internet]. Advisory on the use of Hydroxychloroquin as prophylaxis for SARSCoV2 infection. Accessed on: 23 March 2020. Available from: https://www.mohfw.gov.in/pdf/AdvisoryontheuseofHydroxychloroquinasprophylaxisforSARSCoV2infection

4. Gautret P, Lagier J-C, Parola P, et al. Hydroxychloroquine and azithromycin as a treatment of COVID-19: results of an open label non-randomized clinical trial. Int J Antimicrob Agents 2020; 2020; published online March 20. DOI:10.1016/j.ijantimicag. 2020.105949.

5. Chen Jun, Liu Danping, Liu Li, et al. A pilot study of hydroxychloroquine in treatment of patients with common coronavirus disease-19 (COVID-19). J Zhejiang Univ (Med Sci) , 2020, 49(1): 0-0.

6. Chan-Yeung M, Xu RH. SARS: epidemiology. Respirology. 2003; 8: S9-14.

7. Mackay IM, Arden KE. MERS coronavirus: diagnostics, epidemiology and transmission. Virology Journal. 2015 Dec 22;1 2(1): 222.

8. WHO Director-General's opening remarks at the media briefing on COVID-19- 11 March 2020. https://www.who.int/dg/speeches/detail/who-directorgeneral opening remarks at the media briefing on covid-19-11 March 2020.

9. Yang N, Shen HM. Targeting the Endocytic Pathway and Autophagy Process as a Novel Therapeutic Strategy in COVID-19. Int J Biol Sci . 2020; 16(10): 1724-1731. Published 2020 Mar 15. doi:10.7150/ijbs. 45498 
10. Biot C, Daher W, Chavain N, et al. Design and synthesis of hydroxyferroquine derivatives with antimalarial and antiviral activities. J Med Chem. 2006. 49(9): 2845-9.

11. . Schrezenmeier E, Dörner T. Mechanisms of action of hydroxychloroquine and chloroquine: implications for rheumatology. Nat Rev Rheumatol . 2020;16(3):155-166.

12. Akhavan PS, Su J, Lou W, Gladman DD, Urowitz MB, Fortin PR. The Early Protective Effect of Hydroxychloroquine on the Risk of Cumulative Damage in Patients with Systemic Lupus Erythematosus. J Rheumatol. 2013 Jun 1; 40(6): 831.

13. Mok, C. C., Mak, A. \& Ma, K. M. Bone mineral density in postmenopausal Chinese patients with systemic lupus erythematosus. Lupus. 2005; 14: 106-112.

14. Petri, M. Use of hydroxychloroquine to prevent thrombosis in systemic lupus erythematosus and in antiphospholipid antibody- positive patients. Curr. Rheumatol. Rep.2011;13:77-80.

15. Mauthe M, Orhon I, Rocchi C, et al. Chloroquine inhibits autophagic flux by decreasing autophagosome-lysosome fusion. Autophagy . 2018; 14(8): 1435-1455. doi:10.1080/15548627.2018.1474314

16. Nakamura N, Rabouille C, Watson R, et al. Characterization of a cis-Golgi matrix protein, GM130. J Cell Biol. 1995. December;131(6 Pt 2):1715-1726. PubMed PMID: 8557739

17. Lotteau V, Teyton L, Peleraux A et al. Intracellular transport of class IIMHCmolecules directed by invariant chain. Nature 1990; 348: 600-5.

18. Wu SF, Chang CB, Hsu JM et al. Hydroxychloroquine inhibits CD154 expression in CD4(!) T lymphocytes of systemic lupus erythematosus through NFAT, but not STAT5, signaling. Arthritis Res Ther 2017; 19: 183.

19. van den Borne BE, Dijkmans BA, de Rooij HH et al. Chloroquine and hydroxychloroquineequally affect tumor necrosis factor-alpha, interleukin 6 , and interferon-gamma production by peripheral blood mononuclear cells. J Rheumatol 1997; 24: 55-60

20. Millet JK, Whittaker GR. Host cell proteases: critical determinants of coronavirustropismand pathogenesis. Virus Res 2015; 202: 120-34.

21. . Millet JK, Whittaker GR. Physiological and molecular triggers for SARS CoV membrane fusion and entry into host cells. Virology. 2018; 517: 3-8.

22. Schrezenmeier E, Dorner T. Mechanisms of action of hydroxychloroquine and chloroquine: implications for rheumatology. Nat Rev Rheumatol 2020; 16: 155-66.

23. Al-Bari MAA. Targeting endosomal acidification by chloroquine analogs as a promising strategy for the treatment of emerging viral diseases. Pharmacol Res Perspect 2017; 5: e00293.

24. Vincent MJ, Bergeron E, Benjannet S et al. Chloroquine is a potent inhibitor of SARS coronavirus infection and spread. Virol J 2005; 2: 69

25. Ghislat, G. \& Lawrence, T. Autophagy in dendritic cells. Cell Mol. Immunol. 2018; 15: 944-952.

26. Ireland, J. M. \& Unanue, E. R. Autophagy in antigen- presenting cells results in presentation of citrullinated peptides to CD4 T cells. J. Exp. Med. 2011; 208: 2625-2632.

27. Rebecca, V. W. et al. PPT1 promotes tumor growth and is the molecular target of chloroquine derivatives in cancer. Cancer Discov. 2019; 9: 220-229 .

28. Prentice E, Jerome WG, Yoshimori T, Mizushima N, Denison MR. Coronavirus replication complex formation utilizes components of cellular autophagy. J Biol Chem. 2004; 279: 10136-41.

29. Prentice E, McAuliffe J, Lu X, Subbarao K, Denison MR. Identification and characterization of severe acute respiratory syndrome coronavirus replicase proteins. J Virol. 2004; 78: 9977-86.

30. Cottam EM, Maier HJ, Manifava M, Vaux LC, Chandra-Schoenfelder P, Gerner W, et al. Coronavirus nsp6 proteins generate autophagosomes from the endoplasmic reticulum via an omegasome intermediate. Autophagy. 2011; 7: 1335-47.

31. Snijder EJ, van der Meer Y, Zevenhoven-Dobbe J, Onderwater JJ, van der Meulen J, Koerten HK, et al. Ultrastructure and origin of membrane vesicles associated with the severe acute respiratory syndrome coronavirus replication complex. J Virol. 2006; 80: 5927-40.

32. Zumla A, Chan JF, Azhar EI, Hui DS, Yuen KY. Coronaviruses - drug discovery and therapeutic options. Nat Rev Drug Discov. 2016; 15: 327-47. 
33. de Haan CA, Rottier PJ. Molecular interactions in the assembly of coronaviruses. Adv Virus Res. 2005; 64: 165-230.

34. Ducatelle R, Hoorens J. Significance of lysosomes in the morphogenesis of coronaviruses. Arch Virol. 1984; 79: 1-12.

35. Laaksonen, A. L., Koskiahde, V. \& Juva, K. Dosage of antimalarial drugs for children with juvenile rheumatoid arthritis and systemic lupus erythematosus. A clinical study with determination of serum concentrations of chloroquine and hydroxychloroquine. Scand. J. rheumatol.1974; 3: 103-108.

36. Huang, C. et al. Clinical features of patients infected with 2019 novel coronavirus in Wuhan, China. Lancet. 2020; 395: 497-506.

37. Sun Hee Lee, Hyunjin Son, Kyong Ran Peck, Can postexposure prophylaxis for COVID-19 be considered as one of outbreak response strategies in long-term care hospitals?, International Journal of Antimicrobial Agents.2020. doi:https://doi.org/10.1016/j.ijantimicag.2020.105988

\section{Hosted file}

Figures Insights into CQ and HCQ.docx available at https://authorea.com/users/ 323324/articles/452066-insights-into-the-mechanisms-of-action-of-chloroquine-andhydroxychloroquine-and-its-use-in-covid-19-for-chemoprophylaxis 